\title{
Article
}

\section{Determining the ideal mattress firmness based on anthropometric measurements}

Shore, Hannah, Richards, James and Chohan, Ambreen

Available at http://clok.uclan.ac.uk/31171/

Shore, Hannah, Richards, James ORCID: 0000-0002-4004-3115 and Chohan, Ambreen ORCID: 0000-0003-0544-7832 (2019) Determining the ideal mattress firmness based on anthropometric measurements. Sleep Medicine, 64 (S1). S350. ISSN 1389-9457

It is advisable to refer to the publisher's version if you intend to cite from the work. http://dx.doi.org/10.1016/j.sleep.2019.11.977

For more information about UCLan's research in this area go to

http://www.uclan.ac.uk/researchgroups/ and search for < name of research Group>.

For information about Research generally at UCLan please go to http://www.uclan.ac.uk/research/

All outputs in CLoK are protected by Intellectual Property Rights law, including Copyright law. Copyright, IPR and Moral Rights for the works on this site are retained by the individual authors and/or other copyright owners. Terms and conditions for use of this material are defined in the policies page. 


\title{
Determining The Ideal Mattress Firmness Based on Anthropometric Measurements
}

\author{
Hannah Shore, Jim Richards, Ambreen Chohan \\ Allied Health Research Unit, University of Central Lancashire, Preston, UK
}

Introduction

Mattresses need to provide enough support to keep spinal alignment close to a neutral posture, whilst minimising muscle activity and providing optimum pressure relief. There is limited evidence to suggest that a 'one size fits all' mattress provides the appropriate support for individuals with diverse body shapes, so a greater understanding of how different mattresses affect the human body is key. By having a more objective approach to choosing a mattress an individual may have an improved quality of sleep.

\section{Materials and Methods}

A ten camera infrared movement analysis system recorded the movement of retroreflective markers placed on the Upper-Mid Thoracic, Mid-Lower Thoracic, Lower Thoracic-Upper Lumbar, Upper-Lower Lumbar and Lower Lumbar-Pelvic areas of the spine. A static image of the spine was taken in a standing position and was used to define each individual's neutral posture. Deviations away from this neutral position were assessed under three different conditions in side lying. Three visually identical mattresses were tested, internally each mattress contained a different firmness of spring unit (soft, medium, firm) with an identical gel foam comfort layer. In addition, height, weight, shoulder width and hip circumference measurements were taken to determine differences in body types.

Results

Spinal alignment was assessed on 59 healthy participants and no significant differences were seen between the different mattress configurations. However, further analysis showed significant differences in spinal alignment between the different mattress conditions within different body shape subgroups. Subgroups were defined using body weight, height, BMI, shoulder width and hip circumference. Those with a higher body weight had a more neutral spinal alignment when on a firmer mattress, whereas those with a lower body weight were better suited to a softer mattress. Shorter people were better aligned on a softer mattress, and a medium mattress kept the spine in a more neutral position amongst taller people. There were no differentiating factors between shoulder width or BMI groups. However, those with a larger hip circumference had significantly greater spinal deviations when on a softer mattress, implying that a softer mattress should be avoided by this subgroup.

Conclusion

This study suggests that a 'one size fits all' approach to mattresses may not be appropriate. Contrasting body types need different levels of support to improve overall spinal alignment, allowing the intervertebral disc to rehydrate, and spinal muscles to relax throughout the night. The use of simple anthropometric measurements could make the selection of the most appropriate mattress easier for the general public.

Acknowledgements

This is part of an Innovate UK Knowledge Transfer Partnership between the University of Central Lancashire and Silentnight Group Ltd. 